\title{
Peripheral femoral venoarterial extracorporeal membrane oxygenation as bridge to heart-lung transplant
}

\author{
Erik Orozco-Hernandez ${ }^{1}$, Brett Melnikoff ${ }^{1}$, Megan Lusby ${ }^{1}$, Jose Tallaj $^{1}$, and Charles \\ Hoopes $^{1}$
}

${ }^{1}$ The University of Alabama at Birmingham Hospital

May 8, 2020

\begin{abstract}
Heart-Lung transplant (HLT) is a widely accepted modality for certain patients with advanced and refractory cardiopulmonary disease. Some of these patients are critically ill on the transplant waiting list, and venoarterial extracorporeal membrane oxygenation (VA-ECMO) can be used as a bridge to transplantation. Although the experience with ECMO as a bridge to lung transplant is promising, there is limited evidence to use ECMO as a bridge to HLT. Femoral cannulation remains a concern for ambulation given the risk of bleeding and cannula complications despite studies reporting its safety. We present a case of a 56-year-old male with interstitial lung disease (ILD) and severe secondary pulmonary hypertension, who was successfully bridged to HLT with ambulatory femoral VA-ECMO.
\end{abstract}

\section{Introduction}

The number of heart-lung transplants (HLT) remained static during the last five years, with 59 procedures in 2017. ${ }^{1}$ Currently, most of the HLTs are performed on patients with severe pulmonary hypertension associated with congenital heart disease, followed by idiopathic pulmonary arterial hypertension (IPAH) and cystic fibrosis $(\mathrm{CF})$. There is also a small trend showing an increasing number of HLTs performed for idiopathic interstitial pneumonia (IIP). ${ }^{2}$ Some of these patients are critically ill on the waiting list, and venoarterial extracorporeal membrane oxygenation (VA-ECMO) can be used as a bridge to transplantation. The evidence to support this strategy is limited but evaluation of the outcomes is crucial, especially with the new heart status allocation system. ${ }^{3}$ Traditionally, ambulation was considered too dangerous with femoral cannulation. We present the case of a 56 -year-old male with interstitial lung disease (ILD) and severe secondary pulmonary hypertension, who was successfully bridged to HLT with ambulatory femoral VA-ECMO.

\section{Case presentation}

The patient is a 56-year-old African American male former smoker with rheumatoid arthritis and pulmonary hypertension who was diagnosed with nonspecific interstitial pneumonia in 2011 after a lung biopsy. He required supplemental oxygen at home and was managed with an immunosuppressive regimen including azathioprine and prednisone. In early 2019 he was admitted with symptoms of congestive heart failure. Transthoracic echocardiogram (TTE) indicated a left ventricular ejection fraction (LVEF) of 45-55\% and reduced right ventricular function. Heart catheterization noted an elevated pulmonary artery pressure of 68/31, pulmonary capillary wedge pressure (PCWP) of 23, and Fick cardiac index (CI) of 1.77. Cardiac MRI and TTE displayed pulmonary hypertension with evidence of right heart failure, severe tricuspid regurgitation, and reduced left ventricular function.

Elevated PCWP with depressed biventricular function and CI suggested the need for HLT. While undergoing additional pre-operative workup his clinical status acutely deteriorated despite maximal medical therapy. He was urgently taken to the operating room and successfully placed on peripheral femoral VA-ECMO with 
a percutaneous technique as a bridge to transplant (BTT). We used a 15 fr Bio-Medicus femoral arterial cannula (Medtronic), 27 fr Bio-Medicus venous femoral cannula (Medtronic), and a distal perfusion catheter with a $5 \mathrm{fr}$ sheath (Cordis). The cannulas were connected to a Cardiohelp system (Maquet) with initial flows started at $3.5 \mathrm{~L} / \mathrm{min}$; there was immediate clinical improvement. The cannulas were secured to the skin with several size 0 silk sutures as well as two foley catheter anchoring device for each cannula, preventing any tension or movement.

He was extubated within 12 hours of cannulation. In order to improve and optimize his physical condition, his rehabilitation was focused on nutrition and ambulation A multidisciplinay team composed of perfusion specialists, nurses and physical therapists (PT) guided and monitored the patient during bed mobility, transfers and ambulation. Prior to ambulation, the patient was considered stable in his cardiopulmonary and mental status, without any bleeding and with adequate physical strength and activity tolerance. The cannulated leg was allowed a range of hip flexion from 0-90 degrees given stable ECMO flows. The patient was allowed to sit at the edge of the bed and in a recliner chair with hip flexion no more than 90 degrees as to encouarge posture and upright position throughout the day. Physical exercise included assisted active bed and chair exercises, sitting, standing and walking. Hemodynamics, comfortable respiratory status and safety were the highest priorities. He was standing with PT assistance the day after cannulation, stood for 10 minutes with contact guard assist (CGA), completed pre-gait activites (weight shifting) and sat in the recliner chair. He was also instructed in a seated exercise program. Five days later he needed only minimal assistance to transfer from bed to chair and started ambulating (walked $410 \mathrm{ft}$ with walker and CGA ). Prior to transplant he was able to walk almost $700 \mathrm{ft}$ (Fig.1). Of note, on admission before ECMO cannulation, he was walking just $300 \mathrm{ft}$, limited by shortness of breath. He did not develop any complications related to ambulation.

On ECMO day 11, a suitable donor became available (fig. 2). He was taken to the operating room for heartlung block transplant, ECMO decannulation and femoral vessel repair. The chest was left open secondary to coagulopathy and closed the next day. Postoperatively, he was started our standard immunosuppressive regimen.

He was discharged from the hospital on postoperative day 21. The patient continues to do well, with normal hemodynamics on his most recent echocardiography and mild (1R) rejection on biopsy. He is stable on New York Heart Association functional class I status, exercises on a treadmill twenty minutes five days a week, and has resumed normal physical activity.

\section{Discussion}

HLT is widely accepted for certain patients with advanced and refractory cardiopulmonary disease. From the first surgery in 1968, more than 4000 HLTs have been performed to date. Currently, the median survival is 6.5 years. Most HLTs are performed on patients with severe pulmonary hypertension associated with congenital heart disease, although there is a trend towards more HLT for IIP. ${ }^{1}$

ECMO has been used as a BTT with a high risk of morbidity and mortality. Even as waiting list mortality declined almost $40 \%$ after the introduction of the Lung Allocation Score in 2005, annual waiting list deaths in the United States reach almost 300, with fibrotic disease as the leading cause. ${ }^{9}$ ECMO as a BTT in end-stage lung disease has increased, but still only represents $1.5 \%$ of the total lung transplantation volume. Otherwise, the outcomes of awake ambulatory ECMO and spontaneous breathing can be excellent. ${ }^{4-8}$

Although the experience with ECMO as a bridge to lung transplant is encouraging, there is limited evidence to use ECMO as a bridge to HLT. A previous analysis by Sertic et al reported a $50 \%$ mortality at 30 days and $50 \%$ mortality at 1 year in patients with ECMO before HLT. ECMO was identified as an strong predictor of mortality. ${ }^{3}$

Femoral VA-ECMO support can be associated with deconditioning, muscle wasting and diaphragmatic weakening secondary to the required bedrest born out of concerns for bleeding or cannula dislodgement. Ambulation is crucial to prevent complications, and early mobilization reduces intensive care unit and overall 
hospital length of stay. ${ }^{10-11}$ Multiple studies have reported the feasibility and safety of ambulating patients with femoral cannulas. ${ }^{10,12-13}$

Pasjira et al decribed 15 cases of ambulatory femoral VA-ECMO, just two of them were a bridge to heart transplant. The ECMO cannulation and ambulation protocol is very similar to ours, median time from cannulation to out of bed was three days, time from cannulation to ambulation was four days and the median distance walked on the last day was $300 \mathrm{ft} .{ }^{10}$ Shudo et al reported a case of ambulatory femoral VA-ECMO as bridge to HLT. The patient started to ambulate after day 9 and could ambulate with minimal assistance. ${ }^{12}$ Before ambulation, we performed a careful evaluation of neurological, cardiovascular, respiratory, musculoskeletal and hematologic (no bleeding) systems. Our patient was out of bed on the first day post cannulation, and he increased ambulation distance before transplant.

\section{Conclusions}

HLT is a definitive treatment for end-stage cardiopulmonary failure. In critically ill patients, VA-ECMO can be used to mitigate end-organ dysfunction while awaiting suitable organs. Ambulatory Femoral VAECMO cannulation seems to be safe and effective in carefully selected patients and can mitigate many of the consequences related to ongoing bedrest.

\section{References}

1. Chambers DC, Cherikh WS, Cherikh WS, et al. The Registry of the International Society for Heart and Lung Transplantation: thirty-sixth adult lung and heart-lung transplantation report 2019; focus theme: Donor and Recipient size match. The Journal of Heart and Lung Transplantation, Vol.38, No. 10, October 2019.

2. Chambers DC, Cherikh WS, Goldfarb S, et al. The Registry of the International Society for Heart and Lung Transplantation: thirty-fifth adult heart transplantation report 2018; focus theme: multiorgan transplantation. The Journal of Heart and Lung Transplantation, Vol 37, No 10, October 2018.

3. Sertic F, Crespo MM, Habertheuer A, et al. Early Outcomes with the Use of Extra Corporeal Membrane Oxygenation as a Bridge to Combined Heart and Lung Transplant. The Journal of Heart and Lung Transplantation. 2019. Volume 38, Issue 4, Supplement, Page S55.

4. Aladdein M, Chatterjee S, Loor G. Bridging to Lung Transplantation. Crit Care Clin 35 (2019) 11-25

5. Hakim AH, Ahmad U, McCurry KR, et al .Contemporary Outcomes for Extracorporeal Membrane Oxygenation Used as Bridge to Lung Transplantation. Annals of Thoracic Surgery 2018; 106:192-8.

6. Rehder KJ, Turner DA, Hartwig MG, et al. Active Rehabilitation during Extracorporeal Membrane Oxygenation as a Bridge to Lung Transplantation. Respir Care 2013; 58: 1291-8.

7. Shafii AE, Mason DP, Brown CR, et al. Growing Experience with Extracorporeal Membrane Oxygenation as a Bridge to Lung Transplantation. ASAIO J 2012; 58:526-9.

8. Hoopes CW, Kukreja J, Golden J, Davenport DL, Diaz-Guzman E, Zwischenberger JB. Extracoporeal Membrane Oxygenation as a Bridge to Pulmonary Transplantation. J Thorac Cardiovasc Surg 2013;145:862-7; Discussion 867-8.

9. Egan TM, Edwards LB. Effect of the Lung Allocation Score on Lung Transplantation in the United States. J Heart Lung Transplant 2016;35:433-9.

10. Pasrija C, Mackowick KM, Raithel M, et al. Ambulation with Femoral Arterial Cannulation Can Be Safely Performed on Venoarterial Extracorporeal Membrane Oxygenation. Annals of Thoracic Surgery 2019; 107:1389-94.

11. Schweickert WD,Pohlman MC, Pohlman AS, et al. Early Physical and Occupational Therapy in Mechanically Ventilated, Critically Ill Patients: a randomised controlled trial . Lancet 2009; 373 :1874-82.

12. Shudo Y, Kasinpila P, Lee AM, et al. Ambulating Femoral Venoarterial Extracorporeal Membrane Oxygenation Bridge to Heart-Lung Transplant. J Thorac Cardiovasc Surg 2018;156:e135-7

\section{Figure Legends}

Fig1. Patient ambulating on peripheral VA-ECMO with the nurse and ECMO specialist 
Fig 2. Heart-Lung block

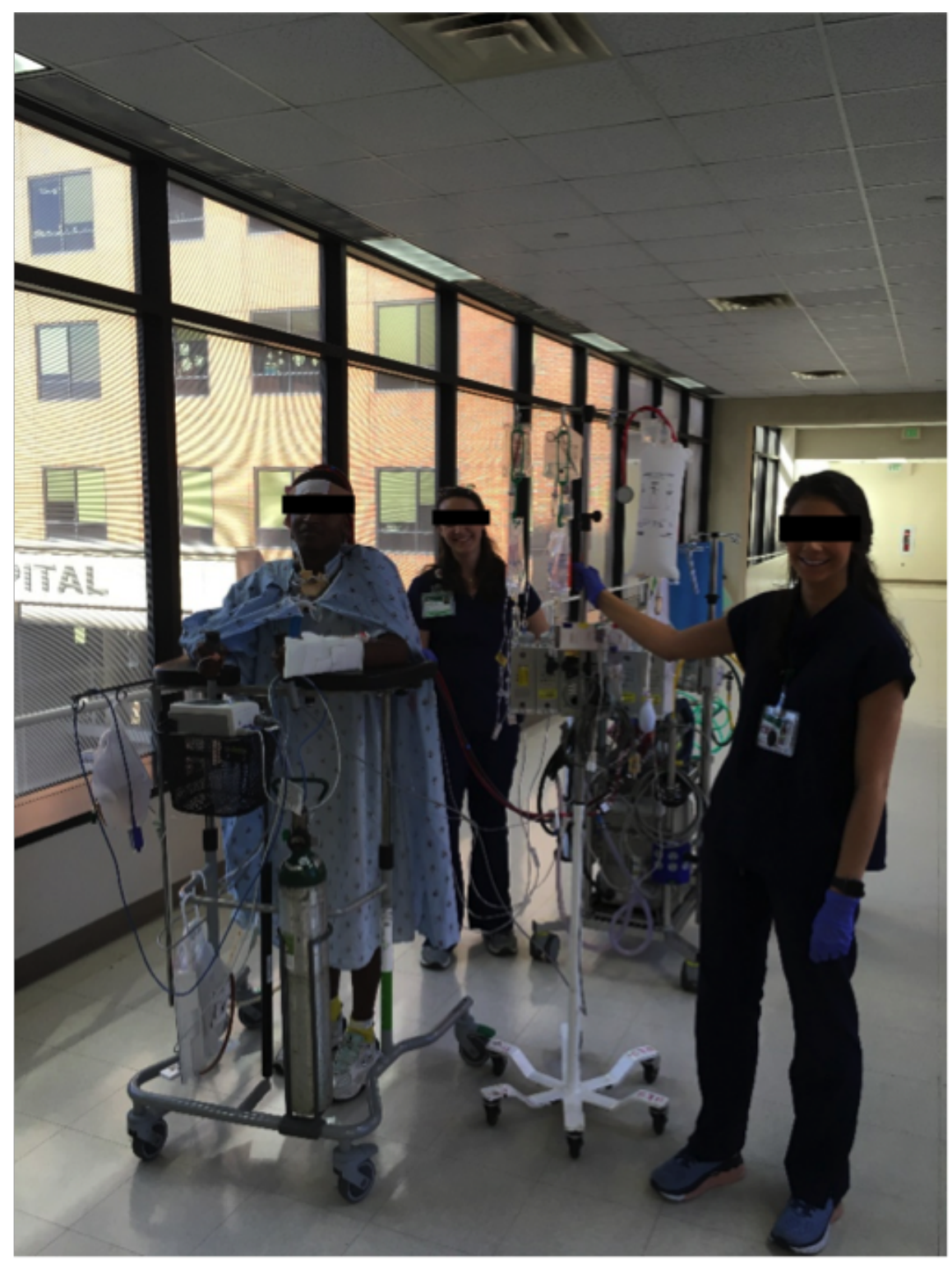




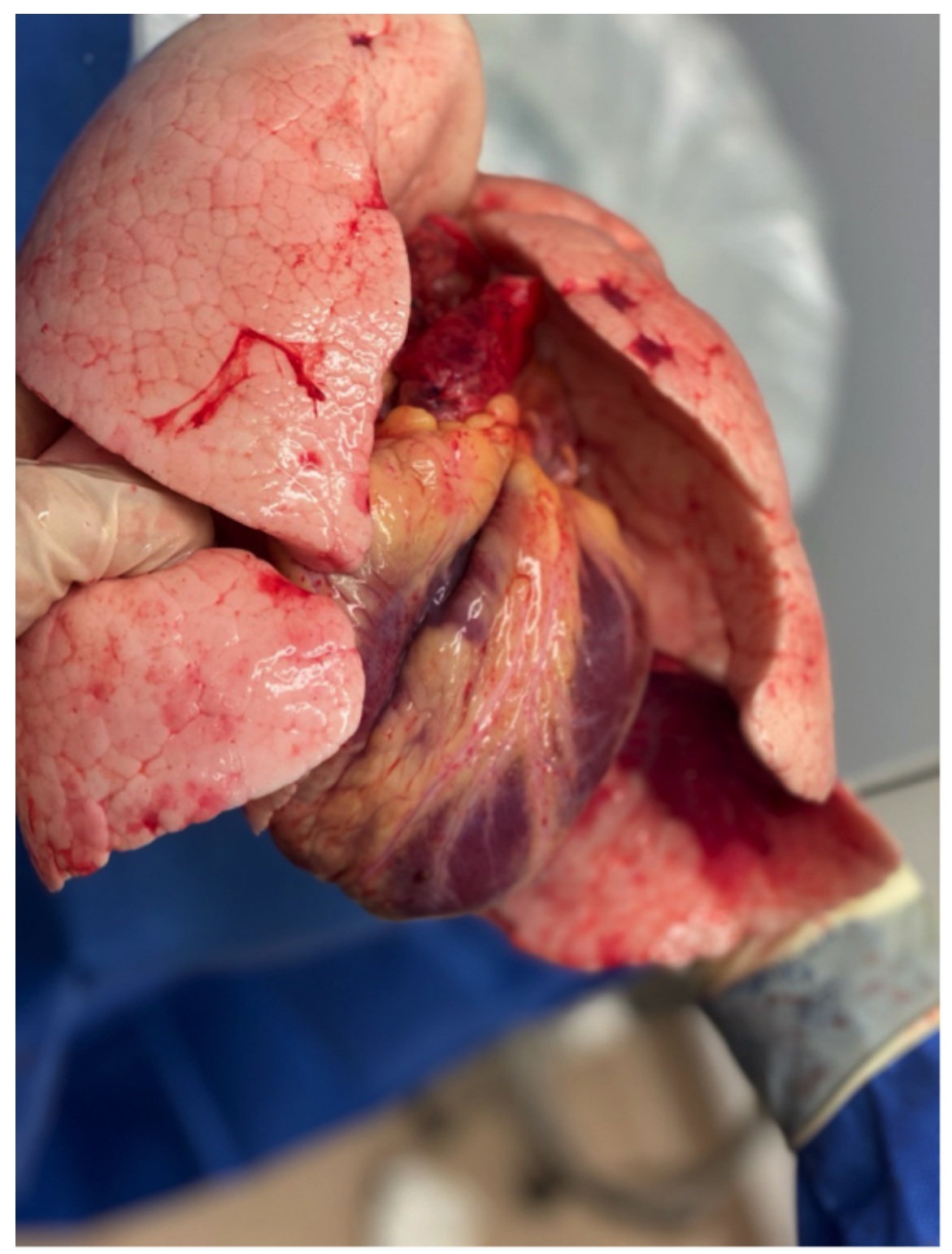

